\title{
ALÉM DA METAFORIZAÇÃO UMA VISÃO BLOCHIANA SOBRE CAOS E FRACTALIDADE*
}

\author{
Rainer E. Zimmermann ${ }^{1}$ \\ Xiaomeng Zhang ${ }^{2}$
}

\begin{abstract}
Resumo
Tendo em vista o projeto em andamento de reinterpretar o conceito blochiano de "obscuridade do instante vivido", serão discutidas as possíveis relações, por um lado, entre as ciências e a filosofia, e, por outro lado, especialmente entre conceitos blochianos e a moderna teoria do caos e fractalidade. O objetivo é demonstrar que categorias científicas podem ser de grande valia quando aplicadas às categorias das ciências humanas (e/ou da filosofia propriamente dita) e podem ultrapassar a mera metaforização de conceitos, como está em voga em muitas áreas. Embora a metaforização certamente seja uma técnica hermenêutica em si mesma, o estabelecimento de aplicações em uma ampla gama de tópicos de pesquisa pode ser aprimorado quando se aproxima o olhar aos aspectos precisos e formalmente corretos envolvidos. Uma abordagem desse tipo, que se concentra nos fundamentos nos quais os conceitos, formais ou hermenêuticos, de fato são construídos, é adequada não apenas para a relação entre a filosofia e as ciências, mas também com vistas às artes. Como resultado, a atitude estética diante do mundo é, no fim das contas, de algum modo complementar à atitude conceitual. Em princípio, portanto, ambas atendem aos mesmos objetivos de orientação em um mundo complexo.
\end{abstract}

Palavras-Chave: Consciência. Estética. Obscuridade do instante vivido. Representações fractais. Sistemas dinâmicos caóticos.

\section{BEYOND METAPHORIZATION A BLOCHIAN VIEW ONTO CHAOS AND FRACTALITY}

\begin{abstract}
With the view to the ongoing project of re-interpreting the Blochian concept of the "darkness of lived immediacy", possible relationships between philosophy and the sciences on the one hand, and in particular, between Blochian concepts and modern chaos theory and fractality on the other, are discussed in some formal detail. The objective is to demonstrate that scientific categories can be helpful when applying them to categories of the humane sciences (and/or philosophy proper) and might lead beyond the mere metaphorization of concepts as it is fashionable today in many fields. Though metaphorization is certainly a hermeneutic technique in its own right, the foundation of applications within a wide range of research topics can be improved when looking more closely at the precise and formally correct aspects involved. Such an approach which is taking care on what ground the concepts, formal or hermeneutic, are actually built, is not only suitable as to the relationship of philosophy and the sciences, but also with a view to the arts. As it turns out, the aesthetical attitude towards the world is somewhat complementary to the conceptual attitude after all. Hence, in principle, do both of them serve the same objectives of orientation within a complex world.
\end{abstract}

Keywords: Aesthetics. Chaotic dynamical systems. Consciousness. Darkness of lived immediacy. Fractal representations.

Traduzido do inglês para o português por Anna Maria Lorenzoni.

1 Doutor em Matemática pela Freie Universität de Berlin (1977); Doutor em Filosofia pela Technische Universität de Berlin (1988); livre-docente, com tese de habilitação sobre a Filosofia da Natureza de Schelling; Professor de Filosofia junto a Faculdade de Estudos Gerais da Universidade de Ciências Aplicadas (UAS) de Munique (1995-2017). E-mail: rainer.zimmermann@hm.edu.

2 Doutora em Filosofia pela Universidade Renmin da China (RUC), junto ao Departamento de Artes Liberais, com tese a respeito de Ernst Bloch (junho de 2019). Desde então, leciona no Departamento de Teoria Literária junto a Faculdade de Artes Liberais da Universidade Normal de Hunan, em Changsha (província de Hunan). E-mail: utopia_1990@163.com. 


\section{Introdução}

Recentemente, um de nós (Zh. X.), propôs uma nova concepção para a abordagem de um dos conceitos básicos da filosofia blochiana, qual seja, a chamada "obscuridade do instante vivido", relativa ao imediatismo individual e inicial da existência subjetiva que serve como ponto de partida para a reflexão em primeira instância. Os detalhes filosóficos já foram discutidos em outras ocasiões ${ }^{3}$. O ponto importante é que temos aqui a oportunidade rara de demonstrar, por um lado, a interação direta da filosofia, e, por outro lado, a das ciências, indo além de um contexto exclusivamente heurístico. Essa concepção certamente é útil também para reconciliar a filosofia blochiana com os conhecimentos recentemente adquiridos no interior da moderna teoria de sistemas emergentes (evolutivos) ${ }^{4}$. Além disso, como o conceito de emergência aponta também para processos que produzem efetivamente novos objetos ou processos dentro de um contexto essencialmente auto organizado, isso tudo ganha relevância também tendo em vista a produção estética.

A motivação para este projeto foi originalmente desencadeada pela pertinente tese de doutorado de Craig Hammond (2012) ${ }^{5}$. A tese teve como objetivo tratar da noção blochiana de utopia abstrata, compensatória, relacionada aos aspectos modernos da teoria do caos e complexidade $^{6}$. Devido às possíveis aplicações dessa abordagem às questões cinematográficas, a tese se mostrou especialmente pertinente para um de nós (R. E. Z), que anteriormente havia se dedicado pormenorizadamente a um trabalho semelhante ${ }^{7}$. Contudo, apesar da diversidade

Para questões fundamentais a respeito do conceito blochiano, cf. ZIMMERMANN, Rainer E; ZHANG, Xiaomeng. Sayable and Unsayable within Lived Immediacy. No prelo pelo Bloch Jahrbuch 2017. Veja-se também ZHANG, Xiaomeng. Information and Meaning in Deterministic Chaos: A Blochian Perspective. In.: Multidisciplinary Digital Publishing Institue Proceedings, Basileia, v. 1, n. 3, 2017, p. 247. DOI:10.3390/IS4SI-2017-04090. (Basicamente, esse artigo se baseia em uma conferência proferida no mesmo ano, IS4SI- 2017, na Chalmers University, Göteborg, em junho de 2017, que era uma versão ampliada de uma outra conferência proferida anteriormente, em abril de 2017, no Bertalanffy Centre, Viena).

4 Cf. ZIMMERMANN, Rainer E. Topoi of Systems: On the Onto-Epistemic Foundations of Matter and Information. Capítulo 6, In: BURGIN, Mark; HOFKIRCHNER, Wolfgang (Ed.). Information Studies and the Quest for Transdisciplinarity. Singapore et al.: World Scientific, 2017, p. 191-214.

5 HAMMOND, Craig A. Towards a Neo-Blochian Theory of Complexity, Hope, and Cinematic Utopia. Tese de Doutorado. Lancaster University 2012. DOI: https://doi.org/10.6084/m9.figshare.1003972.v2. Disponível

<https://figshare.com/articles/Towards_a_Neo_Blochian_Theory_of_Complexity_Hope_and_Cinema em: tic_Utopia/1003972>. Acesso em 20 de agosto de 2017.

6 Cf. ibid., par. 1, p. 15 et seq.

7 RASMI, Mahmoud. Mythopoesis, Aesthetics, and Artistic Creation. Towards a Tautegorical Interpretation of the Cinematic Image. Tese de doutorado, Universidade de Salamanca (Espanha), 2015. (Esse trabalho faz referência à mitologia de Schelling e não à perspectiva blochiana, mas é importante observar que ambas estão intimamente relacionadas uma à outra. Desse modo, a "imediaticidade vivida", no sentido de Bloch, desde o início está diretamente ligada a aspectos mitológicos). Isso foi discutido também em ZIMMERMANN, Rainer E. Hoffnung gegen den Tod. Capítulo 17. In: id. (ed.). Ernst Bloch: Das Prinzip Hoffnung (Klassiker Auslegen 56). Berlin/Boston: de Gruyter, 2017, p. 325-335. 
de passagens esclarecedoras dessa tese ${ }^{8}$, ainda permanece em aberto um problema crucial: quando se fala em mente, consciência, ou qualquer outro campo das ciências humanas em seu conjunto, geralmente os conceitos da teoria do caos são utilizados exclusivamente, em maior ou menor grau, em termos metafóricos. Com efeito, já há algum tempo existe uma longa discussão sobre a legitimidade de se fazê-lo, especialmente quando são introduzidos conceitos matemáticos no interior da filosofia francesa estruturalista ou pós-moderna. No que se refere à teoria do caos e da fractalidade, é bastante óbvio, por exemplo, que a propriedade de autossimilaridade $^{9}$ é mais atrativa quando diz respeito a aspectos da consciência. Nesse sentido, a metaforização teria o potencial de auxiliar a revelar a variedade de significados que são relevantes por si mesmos em um determinado tema. Até aqui, tudo bem.

No entanto, como se nota, não se trata apenas de mera metaforização. Há muito mais do que isso. E esse é o ponto central que gostaríamos de enfatizar aqui: o contexto matemático dos sistemas caóticos (bem como a própria noção de sistema) não é arbitrário e não pode ser aceito ou descartado aleatoriamente, em vez disso, trata-se de uma representação precisa e correta daquilo que realmente ocorre na consciência humana. A razão para isso é dual: em primeiro lugar, a consciência é um fenômeno emergente com respeito aos processos cerebrais reais, sendo o cérebro uma estrutura bioquímica composta. E existe, por sua vez, uma modalidade física na base da consciência que pode ser descrita em termos matemáticos.

Em contrapartida, existe também, e este é o segundo ponto, uma relação genérica entre a filosofia e as ciências derivada principalmente da perspectiva de que a filosofia é uma ciência em si mesma, não obstante lide com o aquilo que todas as demais ciências não lidam: ou seja, com a totalidade mediada daquilo que existe. Sendo assim, a filosofia, não está mais uma vez fazendo o que todas as ciências estão fazendo, porém, ao unificar as diversas perspectivas sobre o mundo, depende de seus resultados. O que devemos fazer, portanto, é voltar às definições originais dos conceitos matemáticos envolvidos (seção 2), então re-traçar suas aplicações no tópico em questão, ou seja, fazendo referência ao cérebro, à mente, e à consciência em seu conjunto (seção 3), e, finalmente, observar quais são as consequências práticas (seção 4).

\section{Sistemas Dinâmicos Caóticos}

8 Uma delas é a discussão especialmente instrutiva de “O corcunda de Notre Dame”, da Disney (1996), ibid., p. 180 et seq.

9 Um fractal é, essencialmente, um conjunto que, sob ampliação, é auto-semelhante (Veja-se ibid., p. 37). 
Por razões práticas, tomamos como ponto de partida uma definição mais convencional de sistemas dinâmicos caóticos, em concordância com o trabalho precedente de Robert Devaney ${ }^{10}$. "Seja V um conjunto. Então f: V $\rightarrow$ V é considerado caótico em V se 1. f possui dependência sensitiva às condições iniciais (fronteiriças); 2. f apresenta transitividade topológica; 3. pontos periódicos são densos em V"11. Desse modo, a ideia é visualizar um mapa caótico como algo essencialmente imprevisível, in-decomponível, mas que possua, entretanto, um aspecto de regularidade. Note-se, a esse propósito, que a in-decomponibilidade nos faz recordar da física quântica, quando se discute sobre de-coerência, devido ao acoplamento permanentemente inseparável entre sistema e ambiente.

Convém recordar as definições básicas que estão implicadas na definição 8.5 supracitada: assim, um mapa $\mathrm{f}: \mathrm{J} \rightarrow \mathrm{J}$ possui dependência sensitiva às condições iniciais, se existe algum $\delta>0$ tal que, para cada $x \in J$ e cada vizinhança $N$ de $x$, existe algum $y \in N$ e algum $\mathrm{n} \geq 0$ tal que o montante absoluto $\left|\mathrm{f}^{\mathrm{n}}(\mathrm{x})-\mathrm{f}^{\mathrm{n}}(\mathrm{y})\right|>\delta^{12}$. Então, pelo menos um dos pontos em cada vizinhança de $\mathrm{x}$ separado por x por pelo menos $\delta$ sob (iteração) auto-dobrável de $\mathrm{f}^{13}$.

Além disso, $\mathrm{f}: \mathrm{J} \rightarrow \mathrm{J}$ é considerado topologicamente transitivo, se, para cada par de conjuntos abertos $U, V \subset J$, houver algum $k>0$ tal que $\mathrm{f}^{\mathrm{k}}(\mathrm{U}) \cap \mathrm{V} \neq 0$. Isso significa que existem pontos que podem passar sob iteração de uma vizinhança arbitrariamente pequena para outra. O sistema, portanto, não pode ser decomposto em dois conjuntos abertos separados que são invariantes sob o mapa. Se um mapa possui uma órbita densa, então com certeza é topologicamente transitivo ${ }^{14}$.

Lembre-se de que um subconjunto $\mathrm{U}$ de $\mathrm{S}$ é denso em $\mathrm{S}$, se $\hat{\mathrm{U}}=\mathrm{S}$, onde $\hat{\mathrm{U}}$ é o fechamento de $\mathrm{U}$. Isso consiste em todos os pontos em $\mathrm{U}$ juntamente a todos os pontos limite de $\mathrm{U}$. (Um ponto $\mathrm{x} \in \mathrm{R}$ é um ponto limite de $\mathrm{S}$, se houver uma sequência de pontos $\mathrm{x}_{\mathrm{n}} \in \mathrm{S}$ convergindo para x. Então S é um conjunto fechado, se contiver todos os seus pontos limite. $\mathrm{O}$ ponto $\mathrm{x}$ é um ponto periódico de período $\mathrm{n}$, se $\mathrm{f}^{\mathrm{n}}(\mathrm{x})=\mathrm{x}$.)

A terminologia do sistema aqui é a original (de fato, apresentada na matemática); o conceito mais recente de "sistema", sendo introduzido um pouco mais tarde, como atualmente é comum no campo dos "sistemas emergentes" e afins, deriva-se dela ${ }^{15}$. Intuitivamente,

10 DEVANEY, Robert L. An Introduction to Chaotic Dynamical Systems. 2nd ed. Redwood City (Cal.) et al.: Addison-Wesley, 1989.

11 Ibid., p. 50 (par.).

12 Cf. ibid., 8.2, p. 49.

13 Iteração não é nada além de auto-composição: em vez de $\mathrm{f}$ o $\mathrm{g}(\mathrm{x})=\mathrm{f}(\mathrm{g}(\mathrm{x}))$, temos $\mathrm{f}$ o $\mathrm{f}(\mathrm{x})=\mathrm{ff}(\mathrm{x}))$, e isso $\mathrm{n}$ vezes: $f^{n}(x)=f$ o $\ldots(n-v e z e s)$ o $f(x)$.

14 Cf. op. cit. 8.1 , p. 49.

15 Cabe aqui fazer uma referência breve a uma definição de sistema recentemente introduzida por um de nós (R.E.Z): "Chamamos sistema uma rede de agentes interagentes que produzem um espaço com um limite bem 
pensamos em um sistema dinâmico como uma equação diferencial do tipo $\mathrm{dx} / \mathrm{dt}=\mathrm{f}(\mathrm{x})$, onde $\mathrm{x}$ pode ser um vetor com muitas entradas. Obviamente, os pontos para os quais $\mathrm{f}(\mathrm{x})=0$ caracterizam o comportamento crítico do sistema e são, portanto, chamados pontos críticos. Embora as equações diferenciais, em sua forma tradicional, não permaneceram no centro da discussão matemática, já que dependem de conceitos de continuidade e diferenciabilidade que raramente são encontrados em processos naturais concretos, elas ainda servem como uma aproximação adequada à descrição dos processos em sua totalidade. Isso se deve principalmente à (co-ordenada) variável tempo t que aparece aqui nessa forma de sistema dinâmico. Assim, a expressão acima fornece fundamentalmente uma taxa de alteração para os estados que um sistema pode ocasionalmente acessar. Esse estado pode ser visualizado como um par (x, $\mathrm{f}(\mathrm{x}))$ em termos bastante gerais. Portanto, dx / dt caracteriza a lei do desdobramento da história de um sistema observável por meio de uma sequência de estados representando uma curva em um estado espacial apropriado ${ }^{16}$. Para sistemas clássicos (cotidianos) em física, basta tratar a variável t como o tempo co-ordenado usual, que pode ser identificado com o tempo do relógio. Se estamos lidando com o campo da consciência, podemos manter esse ponto de vista clássico ao visualizar a consciência como emergente em relação aos processos cerebrais envolvidos, que são, basicamente, processos quânticos ${ }^{17}$.

definido e aberto no sentido da termodinâmica" (Metaphysics of Emergence, Parte 1, 2015, p. 27, referência citada abaixo, n. 28 neste texto.) Ao examinar cuidadosamente essa definição, evidencia-se que um sistema é sempre auto-organizado, porque é produzido por seus agentes que são constituintes do sistema. De fato, é possível inclusive aplicar a teoria dos jogos à interação desses agentes. O único pressuposto necessário é a validade da termodinâmica. Isso esclarece, simultaneamente, dois pontos: um é o fato de que, sendo assim, os sistemas computadorizados não são realmente sistemas, no sentido próprio do termo, pois não são produzidos por agentes que constituem eles próprios. Na melhor das hipóteses, são simulações de um sistema. E isso não é a mesma coisa. O segundo ponto é que a definição de sistema pode ser estendida de maneira direta ao próprio Universo. Em princípio, o Universo é o sistema máximo e, portanto, é auto-organizado e, portanto, processa informações: isso ocorre principalmente porque a definição de um agente remete a Stuart Kauffman, que mais uma vez acopla essa definição à termodinâmica. (Fundamentalmente, então, um agente é ele próprio um sistema capaz de executar pelo menos um ciclo de trabalho termodinâmico.) Pode-se mostrar que isso já é verdade para redes de spin, que são os menores constituintes de espaço e tempo no sentido da física (quântica). Os agentes, aqui, são loops quânticos, de modo que seis deles co-operam para formar uma célula hexagonal da rede de spin. Esta rede está realizando o processamento de informações (quânticas). Portanto, poderíamos argumentar que o Universo, em seu nível mais fundamental, não passa de um computador quântico. O que é imediatamente evidente, portanto, é que a informação não emerge tardiamente no domínio biológico, mas sim que está presente desde o início (do Universo). E, mais do que isso: essa informação processada já é significativa, pois o objetivo desses sistemas fundamentais (sua "meta" ou "propósito") é maximizar a complexidade, de acordo com a quarta lei da termodinâmica de Stuart Kauffman (que estabelece que a evolução ocorre no possível adjacente, isto é, a transição de um estado real para um novo estado possível leva sempre àquelas possibilidades que estão a exatamente um passo da reação).

16 Note-se que esse estado espacial não é necessariamente observável. Muitas vezes, nada mais é do que uma construção matemática abstrata que acaba sendo útil. A observação cotidiana (clássica) dos fenômenos físicos geralmente se relaciona a um estado espacial quadri-dimensional especial o qual denominamos espaço-tempo.

17 Divergimos aqui, portanto, da abordagem de Penrose e Hameroff, os quais identificam de forma direta a consciência com os processos quânticos. Em vez disso, indicamos para o fato de que os seres humanos não possuem sentidos físicos capazes de cognição no domínio quântico. Desse modo, o que observamos por meio da modalidade humana é apenas uma aproximação clássica da média (valor médio) do que realmente existe. 


\section{Caos e fractais na consciência}

Agora chegamos ao ponto importante: a ideia que discutimos à luz do artigo de Zhang Xiaomeng supracitado foi baseada em uma nova relação dos padrões fractais com a imediaticidade do instante vivido, no sentido blochiano. Isso ocorre principalmente porque toda a teoria categorial de Bloch está situada antes do início do desdobramento da hierarquia completa de transformações desse estado inicial de imediaticidade, que, por fim, produz as principais categorias em questão. Ou, em outras palavras: é aqui que a reflexão realmente emerge a partir do imediatismo pré-reflexivo. Antes de começar a visualizar possíveis aplicações dos conceitos envolvidos, é importante lançar alguma luz sobre esse momento inicial. A ideia, então, era a de que o padrão inicial dos processos conscientes, quando representados em um estado espacial abstrato adequado, se tornaria uma composição associativa de pontos brilhantes e escuros, onde os primeiros pensamentos claros (isto é, conceituais) apareceriam como pontos mais brilhantes, dispostos de acordo com um padrão fractal no interior de um fundo fundamentalmente escuro, incluindo algum sombreado entre eles, indicando "zonas de transição". (De fato, qualquer padrão fractal desse tipo servirá para nossos propósitos aqui) ${ }^{18}$.

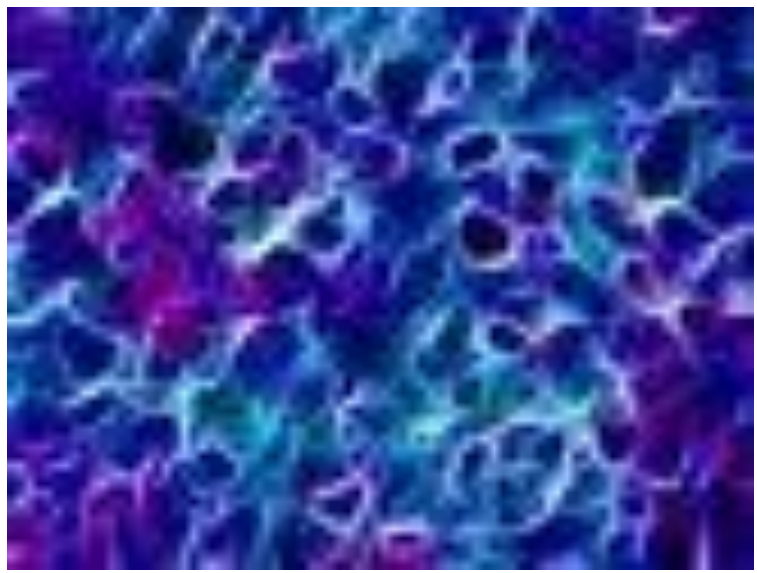

Recorde-se o seguinte: em princípio, as características da obscuridade do instante

18 A figura que se segue, bem como diversas outras do mesmo tipo, pode ser encontrada no seguinte endereço: $<$ https://www.shutterstock.com/video/clip-27822082-stock-footage-abstract-motion-background-in-fieryorange-with-slow-moving-loop-pattern-and-strong-contrast-it.html?src=rel/194554:8> (Acesso em 21-082017). Com efeito, se acessados pela página web, notar-se-á que esses diagramas estão em movimento. Isso os torna ainda mais realistas, pois, obviamente, a estrutura padrão que está em jogo se altera com o tempo. Notese, entretanto, que, quando se fala de "tempo" relacionado a uma consciência individual, isso não significa "tempo relógio", mas uma escala temporal individual (ou: "sistema de temporalidade", no sentido de Prigogine) que está relacionada ao tempo relógio, de modo que este último indica a média convencional obtida a partir de todas essas temporalidades possíveis.

\begin{tabular}{|l|l|l|l|l|}
\hline Q & Anotiala 10 & n. 21 & Janeiro - Abril 2021 & p. $245-257$ \\
\hline
\end{tabular}


vivido blochiana podem ser equiparadas a um estado de completa ausência de contraste. Em outras palavras: ao sair dessa obscuridade, distanciando-se, aplicando as elevações-rotações de Bloch uma após a outra, obtêm-se essencialmente uma uniformidade original dessa obscuridade, que cada vez mais é substituída pelo que podemos chamar contraste.

Geralmente, o contraste é uma propriedade de um sistema de exibição ótica, definido como a proporção da luminescência da cor mais brilhante (branco) e da cor mais escura (preto) que o sistema é capaz de produzir. Nos sistemas de exibição, o objetivo é produzir a maior taxa de contraste possível. Não é por acaso que o conceito de alcance dinâmico está intimamente relacionado a isso. Ele é aplicado a todos os tipos de propriedades de sinais. De modo generalizado, esses sinais podem ser comparados com as ideias nas quais realmente se baseia o processo de pensamento.

Assim, originalmente, o contraste diz respeito à diferença entre as regiões claras e escuras de uma imagem, independentemente de serem realmente regiões concretas ou abstratas (no sentido óptico). Essa significação, portanto, pode ser mantida, se transferirmos toda a expressão para o processo de reflexão: distanciando-se de si mesmo, obtém-se também contraste. E isso significa que o contraste implica forma (formato) - ou seja, aquilo sobre o que se está refletindo. Devemos lembrar que o grafismo humano está implícito em muitas ações cognitivas realizadas ${ }^{19}$. Agora, se pensarmos em uma conexão com o conceito de informação, isso significa que o contraste pode de fato ser visualizado como um medidor para a informação obtida. Como diferimos (dentro do contexto de nossa abordagem sistêmica) entre, por um lado, massa-energia (chamada: matéria) e, por outro lado, estrutura-entropia (chamada: informação), as informações induzidas por esse tipo de contraste se encontram ao lado da estrutura. O que está sendo estruturado aqui, entretanto, não é um espaço comum, mas uma porção individual do espaço social. Podemos chamá-lo de espaço individual de reflexão. O padrão assim alcançado, que é gerado por esse contraste, geralmente tem uma natureza fractal: de fato, até onde podemos enxergar atualmente, muito frequentemente não se trata de um fractal em sentido estrito, pois seria ou uma imagem do espaço fásico ou uma observação concreta do espaço configuracional (euclidiano) (a depender se alguém está falando sobre o caos determinístico no sentido de Mandelbrot e Feigenbaum e, portanto, também Prigogine, ou sobre a criticalidade auto-organizada no sentido da escola de Santa Fe, respectivamente) - e aí ainda não há, até onde podemos ver, nenhuma formalização consistente de espaços de reflexão como espaços fásicos. Mas as propriedades básicas do caos estão efetivamente preenchidas. É nesse sentido que, no

19 A esse respeito, veja-se alguns dos materiais apresentados no artigo sobre "Os Topoi dos Sistemas" mencionado acima. 
diagrama exibido, a coloração (sombreada) se refere a ideias e conceitos, enquanto as manchas escuras são resíduas da bem-conhecida obscuridade do instante vivido - representando a base desestruturada desse espaço de reflexão.

A figura acima, portanto, mostra um adequado (abstrato) estado espacial da consciência e, embora possa não mostrar com precisão um padrão fractal, sua forma associativa provavelmente é clara o suficiente e bastante ilustrativa ${ }^{20}$. Novamente, interessa-nos a autosimilaridade, pois, em certo sentido, os pensamentos podem, sob ampliação, ser visualizados como invariantes, e, uma vez desenvolvidos, em vez de um padrão local, eles tendem a desenvolver um padrão global dentro da consciência, diferentemente do fato de que o processo físico que os está produzindo esteja limitado a uma pequena região do cérebro. É também a estrutura fundamentalmente recursiva dos pensamentos, e sua qualidade não-linear e autocatalítica daí derivada, que sustenta esse tipo de representação. Contudo, como a geometria fractal é apenas um instrumento de representação de processos subjacentes que são deterministicamente caóticos, a questão é se realmente podemos localizar esses processos na concretude física em sua totalidade. Caso contrário, a representação seria apenas formal e arbitrária. Se, entretanto, pudermos localizar tais processos, então sua representação seria mais do que mera metaforização.

Há muito tempo tem se desenvolvido um debate dessa mesma natureza. Uma das primeiras coletâneas de artigos sobre esse assunto foi apresentada já em $1996^{21}$. Em seu prefácio, os editores afirmam que

[...] os fractais podem desempenhar um papel importante na explicação de processos neuronais que geram atividade mental [...] A atividade auto-organizada dos neurônios que reagem ao mundo externo através de percepções e ações produz padrões fractais e do tipo fractal, muitos dos quais são simétricos. [...] a simetria [...] provavelmente ocupa um papel necessário nesse comportamento auto-organizado ${ }^{22}$.

Os editores fazem referência, então, ao trabalho precedente de Walter Freeman ${ }^{23}$. $\mathrm{Na}$ época, contudo, pouco progresso foi efetivamente alcançado, de modo que havia apenas a forte, porém ainda não comprovada, conjectura sobre o papel dos processos caóticos ${ }^{24}$. De todo modo, o caminho era promissor, tendo sempre em mente que “[...] a caracterização da natureza

20 Ao se ampliar a imagem exibida, revela-se uma estrutura ainda mais clara do nível das células constitutivas embora um tanto desfocada, devido à qualidade da resolução alcançada.

21 CORMAC, Earl Mac; STAMENOV, Maxim I. (Ed.). Fractals of brain, fractals of mind. Amsterdam/ Philadelphia: Benjamins, 1996.

22 Ibid., p. ix.

23 Cf. FREEMAN, Walter J. Tutorial on Neurobiology. In: International Journal of Bifurcation and Chaos, v. 2, n. 3, 1992, p. 451-482.

24 Op. cit., p. 1, citando Freeman aqui.

\begin{tabular}{|c|c|c|c|}
\hline Qovista Dialectus & Ano 10 & n. 21 & Janeiro - Abril 2021 \\
\hline
\end{tabular}


da representação é fundamental para responder como podemos ver ou resolver o problema [...]"25. Recentemente, essa ideia foi retomada - como um de nós (REZ) mostrou em um artigo sobre os topoi dos sistemas ${ }^{26}$. Na coletânea de ensaios supracitada, vários autores reunem detalhes explícitos, de modo a apoiar a conjectura original, e o fazem fazendo referência principalmente a trabalhos anteriores sobre auto-organização, teoria do caos e teoria da complexidade. Os fractais são visualizados, aqui, como uma interface computacional formal entre mente e cérebro ${ }^{27}$. Ao mesmo tempo, isso indica para uma mediação de micro-sistemas e macro-sistemas, no sentido físico da física quântica e clássica.

David Alexander e Gordon Globus entendem o caos e a fractalidade como resíduos da complexidade auto-organizada segundo a quarta lei da termodinâmica de Stuart Kauffman (que serve de base para nossa própria visão da dinâmica do sistema conduzido por agentes). É necessário discutir um ou outro problema de maneira mais detalha. Isso, contudo, não poderá ser feito neste $\operatorname{artigo~}^{28}$. Entretanto, quando se referem às obras de Nicolis, Bunge e Langton, os autores revelam sua orientação básica em direção à criticalidade auto-organizada ${ }^{29}$. Carl Anderson e Arnold Mandell, em contrapartida, concentram-se no aspecto do tempo fractal e observam que, o que está sendo discutido atualmente nesse âmbito, teria sido percebido como ruído há apenas uma década (isto é, em meados dos anos 80$)^{30}$. Eles chegaram à conclusão de que

a consciência que evolui em um mundo fractal parece parcimoniosamente exigir a incorporação de estruturas fractais, bem como [...] processos, e estes, por sua vez, seriam integrados a sistemas sensoriais, reconhecimento, memória e comportamento adaptativo. 1/f espectro de potência fractal foi observado em níveis fundamentais de processamento [em uma ampla gama de campos][...] A ubiquidade de 1/f espectro de ruído é nossa evidência para conjecturar [a propriedade de simetrias aproximadas na natureza] $]^{31}$.

Dessa coletânea de ensaios, o que podemos perceber como preponderante, em

25 Ibid., p. 3.

26 World Scientific, 2017, op. cit. - Com efeito, há uma outra questão a esse respeito: os editores se remetem a Smolensky, o qual defende que categorias cognitivas podem ser modeladas como atratores nos sistemas dinâmicos conexionistas (op. cit., p. 5 et seq.). Isso encontra paralelos interessantes com a ideia de Thom, denominada por ele como chreods. A relação explícita com o que está sendo abordado aqui está descrita em outro texto. Veja-se, mais recentemente: ZIMMERMANN, Rainer E. The Return of Metaphysics in the Theory of Subjective Pregnances. In: Proceedings, v. 1, n. 3, 2017. DOI:10.3390/IS4SI-2017-04092.

27 Cf. MAC CORMAC; STAMENOV. Op. cit., p. 8.

28 Cf. p. ex. ZIMMERMANN, Rainer E. Metaphysics of Emergence. Part 1: On the Foundation of Systems. Berlin: Xenomoi, 2015.

29 Alexander, David M.; GLOBUS, Gordon G. Edge-of-Chaos Dynamics in Recursively Organized Neural Systems. In: MAC CORMAC; STAMENOV (Ed.), op. cit., p. 31-73. Veja-se especialmente: p. 37-39.

30 ANDERSON, Carl M.; MANDELL, Arnold J. Fractal Time and the Foundations of Consiousness. In: MAC CORMAC; STAMENOV (Ed.), op. cit., p. 75-126, aqui: p. 75.

31 Ibid., p. 114. 
última instância, é que o pressuposto “"mente’ [...] não é uma substância tradicional diferente do corpo, mas sim parte de um processo físico"32. Nessa perspectiva, "[a] experiência do 'pensamento' é a 'mineração' autocatalítica autorreferencial da organização algorítmica do cérebro" ${ }^{33}$. Larry Vandervert dá um exemplo muito esclarecedor referente à citação de Einstein (“O que, precisamente, é o 'pensamento'?”) das notas autobiográficas deste último, e o faz apontando para um retrato de uma parte do conjunto fractal de Mandelbrot, cujo limite é caracterizado pelos conjuntos de $\mathrm{Julia}^{34}$. O único aspecto um tanto incômodo desse livro - que, por outro lado, é muito esclarecer -, é que muitas vezes a representação do caos no espaço fásico (nos termos da teoria do caos propriamente dita) se confunde com as representações no espaço-tempo observável (criticalidade auto-organizada no sentido da escola de Santa Fé).

Nesse ínterim, nosso conhecimento a respeito do caos e da fractalidade aumentou decisivamente. Um notável estudo a respeito do conhecimento acumulado pode ser encontrado em dois livros de Peitgen e seus colaboradores ${ }^{35}$. Ao ler esse livro, pode se compreender de maneira especial o papel dos conjuntos de Julia como delimitadores fractais de bacias de atração. Quanto à consciência, continua importante o trabalho seminal de Patricia Churchland e Terrence Sejnowski ${ }^{36}$. Embora eles não tratem propriamente sobre caos e fractalidade, emergência e auto-organização são tematizadas topicamente em seu livro ${ }^{37}$.

Mais próximos de nossa temática, estão dois livros mais recentes que apresentam um certo progresso com vistas à possível decisão sobre o papel do caos no cérebro e na consciência. O primeiro é de Christof Koch, o qual afirma, em 1999, que:

\begin{abstract}
pode ser demonstrado analiticamente [...] que grandes redes de neurônios simples, interconectados de maneira esparsa e aleatória com muitas sinapses excitatórias e inibitórias relativamente poderosas, cuja atividade é aproximadamente 'equilibrada', exibem padrões altamente irregulares de picos de atividade característicos do caos determinístico. Essas redes podem responder muito rapidamente ao input externo, muito mais rapidamente do que a constante de tempo dos neurônios singulares. Van Vreeswijk e Sompolinsky (1996) defendem que o pico irregular é uma propriedade de rede emergente robusta que não depende de propriedades celulares complexas. No entanto, essa capacidade de responder rapidamente vem com o preço da atividade continuada, exigindo um gasto constante de energia metabólica ${ }^{38}$.
\end{abstract}

32 Earl R. Mac Cormac: Fractal Thinking: Self-Organizing Brain Processing. In: Mac Cormac, Stamenov (eds.), op. cit., 127-154, aqui: 149.

33 VANDERVERT, Larry. The Fractal Maximum-Power Evolution of Brain, Consciousness, and Mind. In: MAC CORMAC; STAMENOV (Ed.), op. cit., p. 235-271, aqui: p. 256.

34 Ibid., p. 235 et seq.

35 PEITGEN, Heinz-Otto et al. Bausteine des Chaos. Fraktale. Reinbek: Rowohlt, 1998. (Originalmente em inglês, New York: Springer, 1992.) E Id. Chaos. Bausteine der Ordnung. (Nas mesmas cidades, 1998/1992.)

36 CHURCHLAND, Patricia S.; SEJNOWSKI, Terrence J. The Computational Brain. Cambridge (Mass.)/ London: MIT Press, 1992 (5ª impressão, 1999).

37 Ibid., p. 2-3; p. 92-98.

$38 \mathrm{KOCH}$, Christof. Biophysics of Computation. Information Processing in Single Neurons. Oxford University Press, 1999, p. 369. - Aqui o autor faz referência a VAN VREESWIJK, C.; SOMPOLINSKY, H. 
Percebemos que a conexão entre padrões de atividade e representações correspondentes claramente sugere uma possível associação de processos ("pensamento") mentais (consciência) e processos físicos subjacentes no cérebro. Numa coletânea de ensaios ainda mais recente, editada por Jack Tuszynski em $2006^{39}$, isso se torna cada vez mais evidente: apesar de o livro reunir muitas contribuições que estão claramente seguindo o caminho traçado por Penrose e Hameroff, Alwyn Scott, em sua própria contribuição, afirma sem rodeios: “A teoria quântica não é necessária" ${ }^{40}$. Seguramente nós também subscreveríamos essa afirmação. A contribuição de Chris King vai dois passos adiante quando, referindo-se mais uma vez a Walter Freeman, lê-se em dois de seus títulos: "Caos e Dinâmica Fractal como fonte de sensibilidade, imprevisibilidade e incerteza" ${ }^{41}$ e "Excitabilidade caótica e sensibilidade quântica como eucarionte fundador típico" ${ }^{42}$. Quando analisados detalhadamente, esses capítulos mostram um material que pode ser esclarecedor, tendo em conta outros trabalhos recentes sobre os princípios gerais da termodinâmica, estabilidade dimensional fundamentada e transferência de informações em sistemas biológicos ${ }^{43}$.

\section{Conclusões preliminares}

Muito embora Ernst Bloch não esteja lidando com a psicanálise em seus pormenores - diferente de Jean-Paul Sartre que, no início de sua filosofia, baseia seu conceito de "cogito pré-reflexivo" em uma crítica explícita a Freud ${ }^{44}$-, a noção de "obscuridade do instante vivido" é um dos dois conceitos fundamentais que concerne a ambas filosofias: tratase da emergência da reflexão a partir de algo que é essencialmente não-reflexivo. O segundo conceito é o de projeto, que, tanto em Sartre quanto em Bloch, direciona a ação existencial para o futuro. Esses dois conceitos estão na raiz de uma possível identificação das perspectivas de

Chaos in neuronal networks with balanced excitatory and inhibitory activity. In: Science, n. 274, 1996, p. 1724-1726.

39 TUSZYNSKI, Jack A. (Ed.). The Emerging Physics of Consciousness. Berlin et al.: Springer, 2006.

40 SCOTT Alwyn. Physicalism, Chaos, and Reductionism. In: TUSZYNSKI (Ed.), op. cit., p. 171-191, aqui: p. 171 .

41 KING, Chris. Quantum Cosmology and the Hard Problem of the Conscious Brain. In: TUSZYNSKI (Ed.), op. cit., p. 407-456, aqui: 13.8, p. 428.

42 Ibid., 13.11, p. 437.

43 Cf. GRATHOFF, Annette. An Evolutionary View on Function-Based Stability. In: Proceedings, v. 1, n. 3, 2017, p. 54. DOI: 10.3390/IS4SI-2017-03920. Também id.: Stonier's Definition for Kinetic and Structural Information. In: Proceedings Proceedings, v. 1, n. 3, 2017, p. 51. DOI: 10.3390/IS4SI-2017-04012.

44 SARTRE, Jean-Paul. L'Etre et le néant. Paris: Gallimard, 1943. 
Bloch e Sartre no que diz respeito à sua caracterização como "filosofias existenciais" 45 . De fato, com a discussão científica apresentada parcialmente aqui, temos a possibilidade de dar início a uma re-construção moderna da psicanálise. Por um lado, esse é um antigo projeto do próprio Freud, que acabou não emergindo adequadamente devido às revoluções científicas ocorridas por volta de $1900^{46}$. Por outro lado, em seu livro sobre psicanálise, Georg Gfäller (2010) indicou para a necessidade de reformar os fundamentos ontológicos e epistemológicos dessa disciplina, tendo como base pressupostos explicitamente científicos ${ }^{47}$.

Mais uma vez, isso traz consequências para a discussão estética das artes. As artes cinematográficas modernas, particularmente, exibem muitos dos aspectos filosóficos e científicos mencionados acima. Em princípio, esse deve ser o caso para todas as artes, mas algumas delas impõem dificuldades para uma abordagem simples de seus núcleos formais ${ }^{48}$. Fundamentalmente, a ideia é de que, ao se tentar interpretar o significado de seus produtos e contextos, todas essas artes estão sujeitas a uma forma específica de hermenêutica. Mas o núcleo formal de cada hermenêutica tem uma lógica apropriada. Ou, em outras palavras: a matemática está, por um lado, na base da filosofia e da arte, bem como, por outro lado, está na base de todas as ciências (incluindo as ciências humanas e sociais, respectivamente).

Desse modo, a hermenêutica se mostra como uma lógica com informações incompletas, ao passo que a lógica se mostra como uma hermenêutica com informações (quase) completas. Note-se, entretanto, que se trata, de fato, de um eufemismo: isso ocorre principalmente porque a informação (quase) completa na física se deve, antes de tudo, à estrutura comparativamente simples dos sistemas envolvidos. Assim, a completude se mostra como um conceito bastante relativo. No entanto, o quadro metodológico que define um espaço de livre atuação para as teorias e suas aplicações no campo científico é uma rede de regras bastante restrita e precisa, ao passo que essa rede é comparativamente flexível e acessível no caso das artes. Esse é um passo necessário no sentido de apoiar a livre atuação de experimentação inovadora. Afinal, os seres humanos estão permanentemente lidando com a metodologia hermenêutica quando exploram e interpretam o mundo (ou mesmo quando

45 Esse aspecto foi abordado em 1999, durante uma conferência, proferida na UAS de Munique, a respeito das duas filosofias. Cf. ZIMMERMANN, Rainer E.; GRÜN, Klaus-Jürgen (Eds.). Existenz \& Utopie. In: System \& Struktur, v. VII, n. 1 \& 2. Cuxhaven, Dartford: Junghans, 1999.

46 Um compilado bastante expressivo desse projeto, por assim dizer, apócrifo, encontra-se no livro de KITCHER, Patricia. Freud's Dream. A Complete Interdisciplinary Science of Mind. Cambridge (Mass.)/London: MIT Press, 1992.

47 GFÄLLER, Georg R. Die Wirkung des Verborgenen. Unbewußte Hintergründe kommunikativer Prozesse in Unternehmen und Institutionen. Stuttgart: Klett-Cotta, 2010.

48 No caso da música, uma nova abordagem da formalização matemática pode, em última instância, mostrar-se bastante promissora. Cf. MAZZOLA, Guerino. The Topos of Music. Geometric Logic of Concepts, Theory, and Performance. Basel et al.: Birkhäuser, 2002. 
questionam especulativamente o fundamento deste mundo), mas de todo modo precisam confiar no núcleo matemático. Em última instância, trata-se de uma espécie de verdadeira interdisciplinaridade cotidiana.

\begin{tabular}{l|l|l|l|l|}
\hline Q & Ano 10 & n. 21 & Janeiro - Abril 2021 & p. $245-257$ \\
\hline
\end{tabular}

